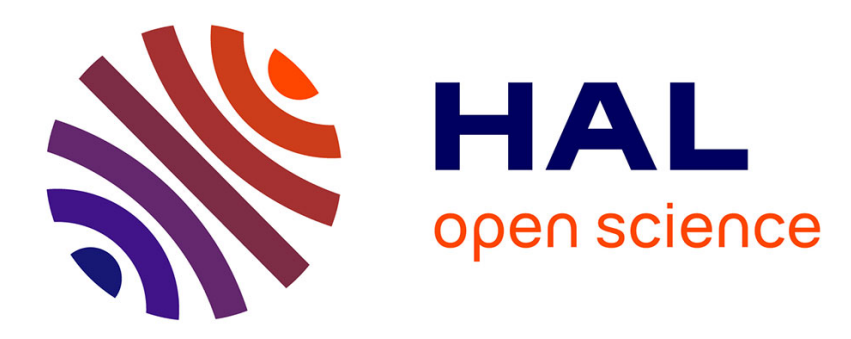

\title{
Support indices: Measuring the effect of input variables over their supports
}

J Fruth, O Roustant, S Kuhnt

\section{To cite this version:}

J Fruth, O Roustant, S Kuhnt. Support indices: Measuring the effect of input variables over their supports. 2018. hal-01113555v4

\section{HAL Id: hal-01113555 \\ https://hal.science/hal-01113555v4}

Preprint submitted on 26 Sep 2018

HAL is a multi-disciplinary open access archive for the deposit and dissemination of scientific research documents, whether they are published or not. The documents may come from teaching and research institutions in France or abroad, or from public or private research centers.
L'archive ouverte pluridisciplinaire HAL, est destinée au dépôt et à la diffusion de documents scientifiques de niveau recherche, publiés ou non, émanant des établissements d'enseignement et de recherche français ou étrangers, des laboratoires publics ou privés. 


\title{
Support indices: Measuring the effect of input variables over their supports
}

\author{
J. Fruth ${ }^{\mathrm{a}, *}$, O. Roustant ${ }^{\mathrm{b}}$, S. Kuhnt ${ }^{\mathrm{a}, \mathrm{c}}$ \\ ${ }^{a}$ Faculty of Statistics, TU Dortmund University, Vogelpothsweg 87, 44227 Dortmund, \\ Germany \\ ${ }^{b}$ Ecole Nationale Supérieure des Mines, FAYOL-EMSE, LSTI, F-42023 Saint-Etienne, \\ France \\ ${ }^{c}$ Dortmund University of Applied Sciences and Arts, Emil-Figge-Strasse 42, 44227 \\ Dortmund, Germany
}

\begin{abstract}
Two new sensitivity indices are presented which give an original solution to the question in sensitivity analysis of how to determine regions within the input space for which the model variation is high. The indices, as functions over the input domain, give insight into the local influence of input variables over the whole domain when the other variables lie in the global domain. They can serve as an informative extension to a standard analysis and in addition are especially helpful in the specification of the input domain, a critical, but often vaguely handled issue in sensitivity analysis. In the usual framework of independent continuous input variables, we present theoretical results that show an asymptotic connection between the presented indices and Sobol' indices, valid for general probability distribution functions. Finally, we show how the indices can be successfully applied on analytical examples and on a real application.
\end{abstract}

Keywords: Sensitivity analysis, Support Analysis, Sobol' indices, DGSM

\footnotetext{
*Corresponding author

Email addresses: fruth@statistik.tu-dortmund.de (J. Fruth), roustant@emse.fr (O. Roustant), sonja.kuhnt@fh-dortmund.de (S. Kuhnt)
} 


\section{Introduction}

Global sensitivity analysis is an essential tool in many of the steps in the analysis of computer experiments, like screening, investigation, model building, or optimization (Saltelli et al., 2000). Variance-based indices have become the method of choice among practitioners, as they offer a clear interpretable quantification of the influence of variables on the output variance. Often a surrogate or meta-model of the computer experiment is build first and indices are derived from this model. In general, different types of variance-based indices have been proposed in the literature. The single influence of a variable is e.g. measured by the first-order Sobol' index (Sobol', 1993). The total sensitivity index (Homma and Saltelli, 1996) quantifies the joint influence of a variable and all its interactions and thus can be used to detect unessential variables. A screening of interactions can be obtained by the total interaction index (Mühlenstädt et al., 2012; Fruth et al., 2014), which captures the total impact of the second-order interaction of a pair of variables.

An alternative concept to global sensitivity are derivative-based global indices (DGSM) (Sobol' and Gershman, 1995; Kucherenko et al., 2009). The indices use the integrated square derivative of the model output over the domain of the inputs as indicator of the input influence over the output and, like total sensitivity indices, can be used to detect unessential variables.

For the estimation of both, variance-based indices and DGSM, input variables are usually treated as random variables following a distribution defined by the user. The minimum and maximum value of the support of this distribution is specified from the region of interest of the parameter in question. They are often chosen vaguely, but can have an important impact on the sensitivity of the response.

As an example, let us consider the Ishigami function by Ishigami and Homma (1990),

$$
f\left(x_{1}, x_{2}, x_{3}\right)=\sin \left(x_{1}\right)+7 \sin ^{2}\left(x_{2}\right)+0.1 x_{3}^{4} \sin \left(x_{1}\right) .
$$

This function is often taken as a benchmark in sensitivity analysis due to its strong non-linearities and interactions. The usual setting considers independent variables $X_{1}, X_{2}, X_{3}$ distributed according to uniform law on $[-\pi, \pi]$, and investigates the Sobol' indices of $f\left(X_{1}, X_{2}, X_{3}\right)$. A direct computation (see e.g. Saltelli et al., 2000) shows that the unnormalized total sensitivity indices relative to $X_{i}$, denoted by $D_{i}^{T}$, of the Ishigami function are equal to 
$D_{1}^{T}=7.72, D_{2}^{T}=6.13$, and $D_{3}^{T}=3.37$. Now, if we reduce the support of the distribution of $X_{3}$ by 10 percent, $X_{3} \sim U\left[-\pi+\frac{\pi}{10}, \pi-\frac{\pi}{10}\right]$, we get $D_{1}^{T}=4.05$, $D_{2}^{T}=6.13$, and $D_{3}^{T}=1.45$. The first and the last variable have rapidly lost influence in comparison to the second one, the ranking of variables has changed. This can be explained by the interaction between $X_{1}$ and $X_{3}$, which is much stronger at the borders than in the rest of the function. The change in the output when changing $X_{2}$ stays the same as $X_{2}$ does not interact with $X_{3}$.

In their book on sensitivity analysis, Saltelli et al. (2000) mention as one goal of sensitivity analysis "to determine the optimal regions within the space of the factors for which the model variation is maximum". We present a method which performs this task by returning the local influences over the whole domain. We extend two scalar indices, namely Sobol' and total sensitivity index. They explore the local sensitivity behavior over the support of the input variables and thus show the impact of the choice of the input distribution.

Our new method is related to the idea of local sensitivities see e.g. Turanyi (1990) for general review on different types of sensitivities. There, local sensitivities are defined as partial derivatives at fixed base-line points. Our method shares with them that partial derivatives are used to obtain local influences. The main difference is that in local sensitivity analysis, the influence of a variable is studied in a local space of the other inputs whereas our method studies the local influence of a variable in a global space of the other variables. The local influences are studied over the whole input space, so no space is ignored. Welch et al. (1992) suggest to plot curves of the main effects, i.e. the expectation of the model conditional on each input, in a framework with a Gaussian process metamodel. They argue that these plots give a visual indication of the effects and provide insight into their relative magnitude. Our idea shares these aims, but gives two different local sensitivities corresponding to first-order Sobol' indices and total indices, which has to our knowledge not been done before.

We next discuss some recent developments towards measuring local or regional sensitivity. Rakovec et al. (2014) suggest a hybrid method which looks at global as well as local sensitivity. They base their approach on gradients at multiple points in the input space. Li et al. (2014) and Wei et al. (2014) consider measures of regional importance which evaluate the impact of inputs with respect to reduced ranges of the individual input space while keeping the input space of the remaining variables global. Similar to our 
method, this results in sensitivity functions over the input space. However, as their measures are not functions directly on the input space but on either a quantile value or the boundaries of the considered interval, we find our new sensitivity indices and plots more intuitive and informative. The approach of Pannier and Graf (2015) follows the same general ideas as our method. They subdivide the input space into a finite number of subdomains and calculate derivative based global sensitivity measures on these subdomains. Our method goes a step further as it can be seen as reducing the discrete subdomains to points of the input space.

With our method, the effects of the choice of the input support do not only become visible, they are also quantified. It gives insight into the function by presenting a function of sensitivities as extension to Sobol' and total sensitivity indices. It can further be a helpful tool in sequential optimization, where the input behavior of certain optimal regions is of interest, which might differ extensively from the global behavior.

After an overview on global sensitivity measures in Section 2, we define the support index functions and show that they are connected to classical indices when the variable in question is restricted to an interval whose size goes to zero (Section 3). Section 4 deals with the estimation of the new support indices. To illustrate the method, it is applied to the Ishigami function, a polynomial function and a real life application in sheet metal forming in Section 5. Conclusions and perspectives are given in Section 6 .

\section{Overview of global sensitivity measures}

We consider a vector $\boldsymbol{X}=\left(X_{1}, \ldots, X_{d}\right)$ of independent random variables with distribution $\mu=\mu_{1} \otimes \cdots \otimes \mu_{d}$, and a black-box function $f$ defined on $\Delta \subseteq \mathbb{R}^{d} \rightarrow \mathbb{R}$ such that $f(\boldsymbol{X}) \in L^{2}(\mu)$. For a given set $I \subseteq\{1, \ldots, d\}$, we denote by $\boldsymbol{X}_{I}$ the vector extracted from $\boldsymbol{X}$ whose coordinates are in $I$.

\subsection{Variance-based sensitivity measures}

The FANOVA decomposition (Hoeffding, 1948; Sobol', 1993) gives a decomposition of the function $f$ into terms of increasing complexity:

$$
f(\boldsymbol{x})=f_{0}+\sum_{i=1}^{d} f_{i}\left(x_{i}\right)+\sum_{1 \leq i<j \leq d} f_{i, j}\left(x_{i}, x_{j}\right)+\cdots+f_{1, \ldots, d}\left(x_{1}, \ldots, x_{d}\right) .
$$


The terms represent first-order effects $\left(f_{i}\left(x_{i}\right)\right)$, second-order interactions $\left(f_{i, j}\left(x_{i}, x_{j}\right)\right)$, and all higher combinations of input variables. Furthermore, when considering the random nature of inputs, the $f_{I}\left(\boldsymbol{X}_{I}\right)$ 's are centered and orthogonal. Thus the variance of $f(\boldsymbol{X})$ can be decomposed accordingly,

$$
\operatorname{var}(f(\boldsymbol{X}))=\sum_{I \subseteq\{1, \ldots, d\}} \operatorname{var}\left(f_{I}\left(\boldsymbol{X}_{I}\right)\right)
$$

where the overall variance is denoted by $D=\operatorname{var}(f(\boldsymbol{X}))$, and the variance terms by $D_{I}=\operatorname{var}\left(f_{I}\left(\boldsymbol{X}_{I}\right)\right)$. These terms quantify the influence of the set of variables $X_{I}$ on the output variance. The Sobol' sensitivity indices $S_{I}$ are defined as the ratio of variance explained by $X_{I}$ divided by the overall variance, ensuring the indices to fall in $[0,1]$ and sum to one

$$
S_{I}=\frac{D_{I}}{D}
$$

In a sensitivity analysis procedure, the first-order Sobol' indices $S_{i}, i=$ $1, \ldots, d$ are usually estimated to quantify the single influences of the input variables. In addition, often the unnormalized and normalized total sensitivity indices

$$
D_{i}^{T}=\sum_{J \supseteq\{i\}} D_{J} \text { and } \quad S_{i}^{T}=\frac{D_{i}^{T}}{D} .
$$

are estimated, which give the influence of each variable including all interactions and thus are suitable for variable screening.

\subsection{Derivative-based measures}

A further sensitivity measure, the derivative-based global sensitivity measures (DGSM), was introduced by Sobol' and Gershman (1995) and studied in Kucherenko et al. (2009). Here, the notion of sensitivity is based on the derivative of the function, rather than on the output variance. It thus summarizes the local variation instead of the global one. The DGSM are defined by

$$
\nu_{i}=\int\left(\frac{\partial f}{\partial x_{i}}(\boldsymbol{x})\right)^{2} d \mu(\boldsymbol{x}) .
$$

It is often convenient to consider a normalized version of $\nu_{i}$,

$$
\nu_{i}^{\star}=\frac{\nu_{i}}{\sum_{j=1}^{d} \nu_{j}} .
$$


Contrary to variance-based indices, DGSM are not associated to a functional decomposition. However they can be connected to total sensitivity indices through

$$
D_{i}^{\mathrm{T}} \leq C\left(\mu_{i}\right) \nu_{i}
$$

provided that $\mu$ belongs to a class of distributions that satisfy a Poincaré inequality,

$$
\int g(\boldsymbol{x})^{2} d \mu(\boldsymbol{x}) \leq C(\mu) \int\|\nabla g(\boldsymbol{x})\|^{2} d \mu(\boldsymbol{x}),
$$

for all functions $g$ in $L^{2}(\mu)$ such that $\int g(\boldsymbol{x}) d \mu(\boldsymbol{x})=0$, and $\|\nabla g\| \in L^{2}(\mu)$, where $\nabla g$ denotes the gradient of $g$ and $C\left(\mu_{i}\right)>0$ constant.

The physical interpretation is that the energy of a centered function, measured by the $L^{2}(\mu)$ norm, is controlled by the energy of its derivative. This explains the Poincaré-type inequality between total Sobol' index and DGSM. Indeed, denote by $g_{i}^{\mathrm{T}}(\boldsymbol{x}):=\sum_{J \supseteq\{i\}} g_{J}\left(\boldsymbol{x}_{J}\right)$ the part of $g(\boldsymbol{x})$ involving $x_{i}$. Then one can check that the energy of $g_{i}^{\mathrm{T}}$ is equal to the total Sobol' index $D_{i}^{T}$ and the energy of its derivative to the DGSM $\nu_{i}$. See Lamboni et al. (2013) or Roustant et al. (2014) for a comprehensive summary. Log concave probability measures fall under this class, thereby most well-known distributions as e.g. uniform, normal and gamma distribution are covered (Lamboni et al., 2013). Equation (6) implies that the DGSM can be used as upper boundaries for the total sensitivity indices. It can thus serve as a cheaper method for the screening of unessential input variables, especially when the computer experiment additionally provides partial derivatives.

\section{Support index functions}

We now derive new sensitivity indices to quantify the changing effect of the input variables over their support. In addition to the previous notation, we assume that $\Delta=[0,1]^{d}, f$ is of class $C^{1}$, and that $X_{1}, \ldots, X_{d}$ have continuous density functions with support $[0,1]$. We will also denote $\boldsymbol{X}_{-i}=$ $\left(X_{1}, \ldots, X_{i-1}, X_{i+1}, \ldots, X_{d}\right)$, and for any function $g$ and any symbol $t$, the notation $g\left(t, \boldsymbol{X}_{-i}\right)$ will assume that $t$ is at position $i$. We are searching for sensitivity measures that quantify the local influence of one variable, including or not its interactions, in the global space of the others. Therefore we restrict $X_{i}$ to vary only over a small interval around $t$ and let the size of the interval go to zero. Proposition 1 summarizes the asymptotic behaviour 
of the overall variance, the Sobol' index and the total sensitivity index of one variable, when the interval size of this variable goes to zero.

Proposition 1. Let $X_{1}, \ldots, X_{d}$ be independent random variables with continuous density functions with support $[0,1]$. Let $f$ be a function defined on $\Delta=[0,1]^{d}$ compact and assume that $f$ is of class $C^{2}$, ensuring that $f(\boldsymbol{X})$, $\frac{\partial f}{\partial x_{\bullet}}(\boldsymbol{X}), \frac{\partial^{2} f}{\partial x_{\bullet} \partial x_{\bullet}}(\boldsymbol{X})$ are in $L^{2}$. Let one of the random variables, say $X_{i}$, be restricted to $X_{i}^{h} \sim X_{i} \mid X_{i} \in[t-h / 2, t+h / 2]$ for $\left.t \in\right] 0,1[$ and $h>0$. Then we have that

$$
\frac{\operatorname{var}\left(X_{i}^{h}-t\right)}{h^{2}} \stackrel{h \rightarrow 0}{\longrightarrow} \frac{1}{12},
$$

and for the limits of the overall variance, the Sobol' index of $X_{i}^{h}$, denoted by $D_{X_{i}^{h}}$, and the total sensitivity index of $X_{i}^{h}$, denoted by $D_{X_{i}^{h}}^{T}$, it holds that

1. $\operatorname{var}\left(f\left(X_{i}^{h}, \mathbf{X}_{-i}\right)\right) \stackrel{h \rightarrow 0}{\longrightarrow} \operatorname{var}\left[f(\mathbf{X}) \mid X_{i}=t\right]$,

2. $\frac{D_{X_{i}^{h}}}{h^{2}}=\frac{\operatorname{var}\left(\mathrm{E}\left[f\left(X_{i}^{h}, \mathbf{X}_{-i}\right) \mid X_{i}^{h}\right]\right)}{h^{2}} \stackrel{h \rightarrow 0}{\longrightarrow} \frac{1}{12}\left(\mathrm{E}\left[\frac{\partial f}{\partial x_{i}}(\mathbf{X}) \mid X_{i}=t\right]\right)^{2}$,

3. $\frac{D_{X_{i}^{h}}^{T}}{h^{2}}=\frac{\mathrm{E}\left(\operatorname{var}\left[f\left(X_{i}^{h}, \mathbf{X}_{-i}\right) \mid \mathbf{X}_{-i}\right]\right)}{h^{2}} \stackrel{h \rightarrow 0}{\longrightarrow} \frac{1}{12} \mathrm{E}\left[\left(\frac{\partial f}{\partial x_{i}}(\mathbf{X})\right)^{2} \mid X_{i}=t\right]$.

The first part of the proposition says that, locally, i.e. restricted to $[t-$ $h / 2, t+h / 2], X_{i}$ has asymptotically the same variance than the uniform distribution, whatever its global distribution $\mu_{i}$. Since that variance equals $h^{2} / 12$, it makes a link to the second part of the proposition, where the term $h^{2} / 12$ appears in the speed of convergence of $D_{X_{i}^{h}}, D_{X_{i}^{h}}^{T}$ when $h$ tends to zero. Notice that the expression of the limits confirm that $D_{X_{i}^{h}}^{T} \geq D_{X_{i}^{h}}$, since the expectation of a square is always greater than the square of an expectation. We base the definition of our new support function on these findings.

Definition 1. Support index functions.

The first-order support index $\zeta_{i}(t)$ of $f(\mathbf{X})$ relative to $X_{i}$ is the squared conditional expectation of its partial derivative with respect to $X_{i}$ :

$$
\zeta_{i}(t)=\left(\mathrm{E}\left[\frac{\partial f}{\partial x_{i}}(\mathbf{X}) \mid X_{i}=t\right]\right)^{2} .
$$


The total support index $\zeta_{i}^{T}(t)$ of $f(\mathbf{X})$ relative to $X_{i}$ is the conditional expectation of its squared partial derivative with respect to $X_{i}$ :

$$
\zeta_{i}^{T}(t)=\mathrm{E}\left[\left(\frac{\partial f}{\partial x_{i}}(\mathbf{X})\right)^{2} \mid X_{i}=t\right] .
$$

Taking the expected value over the support index functions reveals interesting connections to other indices, which give a little more insight into the interpretation of the functions. For the total support index, we immediately get equality to the DGSM index. On the other hand, the expected value over the first-order support index function can be connected to variance-based indices when we replace $f$ as underlying function by the partial derivative of $f$ with respect to $x_{i}$.

Proposition 2. 1 . For the total support index $\zeta_{i}^{T}($.$) and the DGSM \nu_{i}$ (see Eq. (5)) of a variable $X_{i}$, we have

$$
\mathrm{E}\left[\zeta_{i}^{T}\left(X_{i}\right)\right]=\nu_{i}
$$

2. Consider the derivative function $\frac{\partial f}{\partial x_{i}}($.$) with global mean E\left(\frac{\partial f}{\partial x_{i}}(\mathbf{X})\right)$ and denote its first-order Sobol' index with respect to variable $X_{i}$ by $D_{i}\left(\frac{\partial f}{\partial x_{i}}(\mathbf{X})\right)$, then:

$$
E\left[\zeta_{i}\left(X_{i}\right)\right]=D_{i}\left(\frac{\partial f}{\partial x_{i}}(\mathbf{X})\right)+\left(E\left(\frac{\partial f}{\partial x_{i}}(\mathbf{X})\right)\right)^{2} .
$$

Remark 1. Notice that the second point can be connected to Iooss et al. (2012), who also apply Sobol' indices of partial derivatives of $f$. They use the index of $X_{i}$ of the derivative w.r.t to $X_{j}$ as indicator for the interaction effect between $X_{i}$ and $X_{j}$ and the index of $X_{i}$ of the derivative w.r.t to $X_{i}$ as indicator of the nonlinear effect of $X_{i}$.

From the first point, it follows that the expected value of the total support index serves as an upper bound for the total sensitivity index,

$$
D_{i}^{T} \leq C\left(\mu_{i}\right) \mathrm{E}\left[\zeta_{i}^{T}\left(X_{i}\right)\right]
$$

with $C\left(\mu_{i}\right)$ the Poincaré constant of $\mu_{i}$ as before. 
Prop. 2 suggests a simple normalization of support indices. Indeed, it may be useful to compare the support indices both to the global value on the support and to other input variables. Furthermore, a same quantity should be used for first-order and total support indices. Hence, since $\zeta_{i}(t) \leq \zeta_{i}^{T}(t)$, we suggest to normalize $\zeta_{i}(t), \zeta_{i}^{T}(t)$ by the quantity $\sum_{j=1}^{d} \int \zeta_{j}^{T}(s) \mu_{j}(d s)$, which is equal to the sum of DGSMs (Prop. 2): $\sum_{j=1}^{d} \nu_{j}$.

Definition 2. Normalized support index functions.

Let normalized first-order and total support index functions relative to $X_{i}$ be defined as

$$
\zeta_{i}^{\star}(t)=\frac{\zeta_{i}(t)}{\sum_{j=1}^{d} \nu_{j}}, \quad \zeta_{i}^{\star T}(t)=\frac{\zeta_{i}^{T}(t)}{\sum_{j=1}^{d} \nu_{j}} .
$$

This normalization extends in our continuous setting the normalization proposed in Pannier and Graf (2015) for the sectional global sensitivity measure (Eq. 14), where distribution supports are divided in a finite number of intervals. However, contrary to the discrete setting, the normalized support indices do not lie in $[0,1]$, but only their global means do:

$$
0 \leq \mathrm{E}\left(\zeta_{i}^{\star}\left(X_{i}\right)\right) \leq \mathrm{E}\left(\zeta_{i}^{\star T}\left(X_{i}\right)\right) \leq 1
$$

Similarly, the expectation of normalized total support indices sum to 1:

$$
\mathrm{E}\left(\sum_{i=1}^{d} \zeta_{i}^{\star T}\left(X_{i}\right)\right)=1
$$

\section{Estimation of support indices}

In practice, we do not know $f$ and have only access to a sample of input variables $X=\left(\mathbf{X}^{(n)}\right)_{1 \leq n \leq N}$ and corresponding function evaluations $Y=$ $\left(f\left(\mathbf{X}^{(n)}\right)\right)_{1 \leq n \leq N}$. An important question is then to estimate the whole set of support indices $\zeta_{i}(),. \zeta_{i}^{T}($.$) for 1 \leq i \leq d$, at a reasonable cost.

Up to square and square-root transformations, support indices can be viewed as regression curves of partial derivatives $\frac{\partial f}{d x_{i}}(\mathbf{X})$ with respect to input variables $X_{i}$. Notice that the same input variable $X_{i}$ serves as a reference both for differentiation and conditioning. Estimators can then be defined on the diagonal scatterplots $\left(X_{i}, \frac{\partial f(\mathbf{X})}{\partial x_{i}}\right)$ of the gradient of $f$, relying on the usual estimators for conditional estimations, based on smoothing or nonparametric estimation (see e.g. Hastie and Tibshirani, 1990). In the sequel 
we denote by $\widehat{m}_{X, Y}(t)$ such an estimator of $E(Y \mid X=t)$. We now give an algorithm for support indices estimation.

For $i=1, \ldots, d$, do:

1. Compute finite differences of $f$ at sample points: Given a small $h>0$, compute the vector $\mathbf{Z}_{i}=\left(\frac{f\left(X_{1}^{(n)}, \ldots, X_{i}^{(n)}+h, \ldots, X_{d}^{(n)}\right)-f\left(\mathbf{X}^{(n)}\right)}{h}\right)_{1 \leq n \leq N}$

2. Compute the estimators for support indices from conditional expectation estimators: $\widehat{\zeta}_{i}(t)=\left(\widehat{m}_{\mathbf{X}_{i}, \mathbf{Z}_{i}}(t)\right)^{2}, \quad \widehat{\zeta}_{i}^{T}(t)=\widehat{m}_{\mathbf{X}_{i},\left(\mathbf{Z}_{i}\right)^{2}}(t)$.

3. Compute the empirical estimate of DGSMs: $\widehat{\nu}_{i}=\overline{\mathbf{Z}_{i}^{2}}$

Finally, deduce the estimates for normalized support indices by dividing $\widehat{\zeta}_{i}(t), \widehat{\zeta}_{i}^{T}(t)$ by $\sum_{j=1}^{d} \hat{\nu}_{j}$, for $i=1, \ldots, d$.

The first step can be skipped if the gradient is supplied at the same time as the function is evaluated. Assuming that the most important cost is function evaluation (possibly including gradient evaluation if supplied), we then have the following result:

Proposition 3. The whole set of first-order and total support indices, with and without normalization, can be estimated at the cost of $N(d+1)$ function evaluations, and only $N$ function evaluations if the gradient of $f$ is supplied.

Notice that the computational cost for estimating the support indices is comparable to the cost of $N(d+2)$ given in (Saltelli, 2002, Theorem 1) for estimating the whole set of usual first-order and total Sobol' indices.

Remark 2. An alternative for estimating the (unnormalized) support indices functions $\zeta_{i}($.$) 's is to use the fact that if f$ is $C^{1}$ on $[0,1]^{d}$ then

$$
\zeta_{i}(t)=\left(\frac{\partial}{\partial x_{i}} \mathrm{E}\left[f(\mathbf{X}) \mid X_{i}=t\right]\right)^{2} .
$$

Thus an estimate is obtained by numerical derivation from the regression curve of $f(\mathbf{X})$ with respect to $X_{i}$, avoiding to compute the gradient of $f$. However, such simplification seems possible neither for estimating the total support indices $\zeta_{i}^{T}($.$) 's nor for estimating the DGSM \nu_{i}$ 's. 


\section{Examples and application}

We now look at a polynomial function and the Ishigami function from the introduction to show how the new indices capture the changing impact of the variable over the support. Then we give a complete sensitivity analysis of a real-life application in the field of sheet metal forming.

\subsection{A polynomial function}

Let us consider the second order polynomial

$$
f\left(x_{1}, x_{2}, x_{3}\right)=x_{2}-x_{3}^{2} .
$$

For sensitivity analysis, we consider independent random variables $X_{1}$, $X_{2}, X_{3}$ drawn uniformly on $[-1,1]$. Clearly $X_{1}$ is not influential, $X_{2}$ has a linear effect whereas $X_{3}$ has a quadratic linear effect. This behaviour is easily discovered on scatterplots of $f$ (Figure 1 ).

The results of the support analysis are then shown in Figure 2. A 5000-point sample was used, although analytical computations are easy here. More precisely, normalized support curves $\zeta_{i}^{\star}($.$) and total support curves \zeta_{i}^{\star T}$ (.) are represented for $i=1,2,3$. For each $X_{i}$, the overall mean of the latter is also represented, as it is equal to the normalized DGSM $\nu_{i}^{\star}$ (Prop. 2) and can be easily interpreted. In particular $\sum_{i=1}^{d} \nu_{i}^{\star}=1$.

We rediscover on Figure 2 that $X_{1}$ has neither a global nor a local influence as well as the intuition that the location of $X_{2}$ has no impact on its influence to the output. This is explained by its pure linear effect on the output and the uniform distribution over the support. Similarly, $X_{3}$ is all the more influent than closer to the support boundary, explained by its pure quadratic effect and the symmetry with respect to the support. This information seems not easily seen on the usual visualization tools such as scatterplots of Figure 1. Actually, as proved in Prop. 1, this requires an estimate of the derivatives of $f$, which was used to create the new figure.

[Figure 1 about here.]

[Figure 2 about here.] 


\subsection{Ishigami function}

We continue the introductive example about Ishigami function:

$$
f\left(x_{1}, x_{2}, x_{3}\right)=\sin \left(x_{1}\right)+7 \sin ^{2}\left(x_{2}\right)+0.1 x_{3}^{4} \sin \left(x_{1}\right) .
$$

For sensitivity analysis, we consider independent random variables $X_{1}, X_{2}, X_{3}$ drawn uniformly on $[-\pi, \pi]$. First, some elements of a standard global sensitivity analysis are shown in Table 1 and Figure 3, including Sobol' indices and DGSM values, as well as a plot of main effects. We then show the new support index functions in Figure 4.

The results of support analysis confirm the effect observed in the Introduction, that the location of $X_{3}$ on its support has a large influence on its total Sobol' index. Indeed, looking at the (normalized) total support curve $\zeta_{3}^{\star T}($.$) , we can see that this influence is neglectible at the middle of the sup-$ port and increasing when $X_{3}$ gets closer to the boundary. We also see that this behaviour is entirely due to interactions, since the support index values $\zeta_{3}^{\star}(t)$ are null. Beside this the sinusoidal behavior of $X_{1}$ and $X_{2}$ can be observed. As the calculation of Sobol' indices over subdomains in Pannier and Graf (2015) is along the same line as our approach, their Figure 11 for the Ishigami function shows the same general behaviour. However, it consists of a piecewise constant function corresponding to fixed size intervals. In our Figure, we directly obtain a smooth function corresponding to infinitesimal intervals, which gives a more local information.

Surprisingly here, similar insight can be viewed by looking at the variability of the projected points on the scatterplots of $f$ (Figure 3 ). This is due to the fact that, for Ishigami function, the scatterplots of $f$ have common shapes

with diagonal scatterplots $\left(X_{i}, \frac{\partial f(\mathbf{X})}{\partial x_{i}}\right)$ of the gradient of $f$ (Figure 5), on which the support indices are estimated. Actually the conditional variance, visible in the scatterplots of $f$, is the limit of the overall variance when the support length tends to zero, as proved in Theorem 1 . This is much different from the asymptotic behaviour of Sobol' indices, measured by support index functions. In general, scatterplots of $f$ cannot be used to deduce all the information concerning support analysis.

[Table 1 about here.]

[Figure 3 about here.] 
[Figure 4 about here.]

[Figure 5 about here.]

\subsection{Application to sheet metal forming}

The industrial process of deep drawing is a fundamental procedure for forming sheet metal into desired shapes. It is for example extensively applied in the automotive industry in the production of car bodies. In the deep drawing process, a flat sheet metal is pressed with a punch into a die while so-called blankholder keep the metal fixed at the metal borders. More details on the finite element model for the sheet metal forming process can be found in ul Hassan et al. (2013), where the focus lies on reducing springback. The current application aims at analyzing the influence of several input parameters on the thickness reduction of the formed part. A high thickness reduction at a region of the sheet metal means a strong local thinning, which can lead to structural deformations or actual tearing. It is thus of interest to know, which parameters influence the thinning and in which way as well as modeling and optimization.

Eight input variables are varied in this study, listed in Table 2 together with ranges assumed by the practitioners. The four variables flow stress, initial sheet thickness, hardening exponent and sheet layout concern the material. On the other hand, the two variables blankholder force and friction can be varied during the process. In practice, friction can be changed during the process by adding or removing lubricant with high pressure air and oil removing agents. To roughly integrate this change of friction over the process time, friction is modeled as three independent variables corresponding to three stages of the process time over which friction is kept constant. The output value is the maximal thickness reduction, that is the thickness reduction of the point of the formed part with the strongest thinning. It is given as the ratio between thickness reduction and initial thickness which leads to a scalar value between 0 and 1 .

[Table 2 about here.]

As the duration of the simulation lies between two and six hours per run, it is out of the scope of a direct analysis. A common approach to reduce the number of evaluations is to replace the original exact model by a fastto-evaluate metamodel, which is fitted on a comparatively small amount of 
evaluations (see e.g. Fang et al., 2006). For the design of these evaluations, space-filling designs like Latin hypercube design (McKay et al., 1979) are well suited. In this application, a maximin Latin hypercube design with 50 runs is applied. The input variables are scaled to lie between 0 and 1 . While this does not affect the sensitivity analysis, it simplifies computations and is necessary for the comparisons in the support analysis. On the results, a Gaussian process (GP) metamodel with a standard product kernel, a single constant trend, and Matérn 5/2 covariance functions is fitted (see e.g. Santner et al., 2003; Roustant et al., 2012). The accuracy of the metamodel is validated by leave-one-out cross-validation. The root mean square error of the cross-validated predictions equals 0.11 , which is regarded sufficient by the mechanical engineers.

Using the obtained GP metamodel, first-order Sobol' and total sensitivity indices, are estimated by applying independent uniform distributions to the input variables. For the computation and visualization the $\mathrm{R}$ package sensitivity (Pujol et al., 2014) is used.

Variable $X_{8}$, the sheet layout, has the strongest influence followed by the two last parts of the friction $X_{5}$ and $X_{6}$. The total sensitivity indices show strong interactions for those variables. Global sensitivity values for these three variables are provided in Table 3. Notice that the superiority of $X_{8}$ is accentuated when measured by DGSM. This is due to the larger fluctuations of that variable, as can be seen on the scatterplots of main effects (Figure 6).

[Table 3 about here.]

[Figure 6 about here.]

As we are working with the very fast-to-evaluate GP metamodel, we can explore this main effect analysis further by looking at the support indices of the three most influential variables $X_{5}, X_{6}$ and $X_{8}$. This analysis shows us the local influence of the variables over the domain and can in particular check if the chosen input ranges influence the results critically. Support indices are estimated with a 2000 -point sample. The resulting support functions are drawn in Figure 7. The plots show that the chosen ranges indeed seem appropriate, as the output variation is not strong at the borders of the input supports. Clearly $X_{8}$ has a much more local influence. This is not surprising since the mean of the total support curve is equal to the normalized DGSM value, which is much more important for $X_{8}$. The wavy shape of the support 
index function of that variable reveals strong nonlinearities in the relation between this variable and the thickness reduction. For instance, the local influence of $X_{8}$ - when $X_{5}, X_{6}$ lie in $[0,1]$ - is strong around 0.2 and 0.8 , but vanishes around $0.4,0.6$, and 0.9 . The two friction parameters $X_{5}$ and $X_{6}$ show rather similar support functions, underlining that both describe the same type of parameter. The interactions seem to be rather equally spread over the support.

[Figure 7 about here.]

\section{Conclusions and perspectives}

Two new sensitivity indices, the first-order support index and the total support index, have been presented that extend the Sobol' index and the total sensitivity index, respectively. They give an original and global solution to one of the challenges of sensitivity analysis named in Saltelli et al. (2000): to determine regions within the input space for which the model variation is maximum. By returning a function of sensitivities over the input space, the indices give insight into the first-order and the total local behavior of input variables.

On the theoretical level, we showed that the two support index functions are asymptotically connected to their corresponding scalar indices when the support approaches zero. Furthermore, we showed that the expected value over the support of the functions is linked to derivative-based indices and variance-based indices, respectively. The results are general as they only require independence and work for every probability density functions of the input variables.

The functions give insight into the local influence of variables and thus can be used as an extension to a sensitivity analysis via standard scalar indices. They can be especially helpful in the specification of the input distribution, a possibly highly influential, but often vaguely determined point in sensitivity analysis. They can further be useful in optimization to explore potential regions in the input domain.

The estimation of the whole set of first-order and total support indices can be done at a reasonable cost with non-parametric smoothing techniques applied to scatterplots of the output gradient. Indeed, using a Monte Carlo sample of size $N$, the computational cost is equal to $N(d+1)$ where $d$ is the number of input variables, which is comparable to the cost for estimating 
Sobol' indices. Furthermore the cost can be reduced to $N$ when the gradient is known. The accuracy of the estimators directly depends on the accuracy of the smoothers used to estimate a regression curve. This could be enhanced in future works by adapting specific smoothing techniques involving derivatives, known as Average Derivative Estimation (see e.g. Lu, 1996). Finally notice that the normalization proposed in Definition 2 was used by Touzani and Busby (2014) in the framework of DGSMs, who empirically showed its benefit in estimation accuracy. One may wonder whether this remains true for support indices.

A limitation of support indices corresponds to the one observed for the Sobol' indices and derivative-based Sobol' measures. As the support indices represent functions of partial derivatives, strongly varying models will lead to higher support indices than smooth ones, an effect which is not reflected in the scalar indices which just summarize the output variance. This is due to the focus on local sensitivities and only important in the case of strongly varying models, in which careful interpretation is necessary.

Among future works, it would be useful to extend the approach to the case of dependent inputs, and to consider developments for Gaussian process metamodelling linked to derivatives and model error.

\section{Acknowledgements}

This paper is based on investigations of the collaborative research centre SFB 708, project C3. The authors would like to thank the Deutsche Forschungsgemeinschaft (DFG) for funding.

\section{Appendix A. Proofs}

Proof of Proposition 1. Let us first show that

$$
\frac{\operatorname{var}\left(X_{i}^{h}-t\right)}{h^{2}} \stackrel{h \rightarrow 0}{\longrightarrow} \frac{1}{12} .
$$

Let $g$ be the density function of $X_{i}-t, g(x)=f_{X_{i}}(x+t)$. By assumption, $g$ is continuous in $0, g(0)>0$. Consider the density of the truncated variable $X_{i}^{h}-t, g_{h}(x)=\frac{g(x)}{\int_{-\frac{h}{2}}^{\frac{h}{2}} g(t) d t} 1_{\left[-\frac{h}{2}, \frac{h}{2}\right]}(x)$. 
Let $\ell_{h}=\inf _{x \in\left[-\frac{h}{2}, \frac{h}{2}\right]} g(x)$ and $u_{h}=\sup _{x \in\left[-\frac{h}{2}, \frac{h}{2}\right]} g(x)$. With continuity of $f$ in 0 , we have $u_{h} \stackrel{h \rightarrow 0}{\longrightarrow} g(0)$ and $\ell_{h} \stackrel{h \rightarrow 0}{\longrightarrow} g(0)$. By standard integral computations we get

$$
h \ell_{h} \leq \int_{-\frac{h}{2}}^{\frac{h}{2}} g(x) d x \leq h u_{h} \text { and } \frac{h^{3}}{12} \ell_{h} \leq \int_{-\frac{h}{2}}^{\frac{h}{2}} g(x) x^{2} d x \leq \frac{h^{3}}{12} u_{h}
$$

so that for $E\left(\left(X_{i}^{h}-t\right)^{2}\right)=\frac{\int_{-\frac{h}{2}}^{\frac{h}{2}} g(x) x^{2} d x}{\int_{-\frac{h}{2}}^{\frac{h}{2}} g(x) d x}$ it holds that

$$
\frac{\ell_{h}}{u_{h}} \frac{h^{3} / 12}{h} \leq E\left(\left(X_{i}^{h}-t\right)^{2}\right) \leq \frac{u_{h}}{\ell_{h}} \frac{h^{3} / 12}{h} .
$$

By dividing by $h^{2}$ this leads to

$$
\frac{E\left(\left(X_{i}^{h}-t\right)^{2}\right)}{h^{2}} \stackrel{h \rightarrow 0}{\longrightarrow} \frac{1}{12}
$$

It remains to show that $\frac{E\left(X_{i}^{h}-t\right)}{h} \stackrel{h \rightarrow 0}{\longrightarrow} 0$. As

$$
\int_{-\frac{h}{2}}^{\frac{h}{2}} g(x) x d x=\int_{0}^{\frac{h}{2}}(g(x)-g(-x)) x d x
$$

it holds that

$$
\begin{aligned}
\left|E\left(X_{i}^{h}-t\right)\right| & \leq \frac{1}{\ell_{h} h} \int_{0}^{\frac{h}{2}}|g(x)-g(-x)| x d x \leq \frac{1}{\ell_{h} h} \int_{0}^{\frac{h}{2}}\left(u_{h}-\ell_{h}\right) x d x \\
& =\frac{u_{h}-\ell_{h}}{\ell_{h} h} \int_{0}^{\frac{h}{2}} x d x=\frac{u_{h}-\ell_{h}}{\ell_{h} h} \frac{h^{2}}{8} .
\end{aligned}
$$

Thus

$$
\frac{\left|E\left(X_{i}^{h}-t\right)\right|}{h} \leq \frac{u_{h}-\ell_{h}}{8 \ell_{h}} \stackrel{h \rightarrow 0}{\longrightarrow} 0 .
$$

In the following, denote $B_{i}=\frac{X_{i}^{h}-t}{h}$. Remark that $B_{i}$ is bounded by $\left|B_{i}\right| \leq \frac{1}{2}$ and that $\operatorname{var}\left(B_{i}\right) \stackrel{h \rightarrow 0}{\longrightarrow} \frac{1}{12}$. 
Proof of the first statement Write the mean value theorem between a real number $x_{i} \in[t-h / 2, t+h / 2] \subseteq[0,1]$ and $t$

$$
f(\boldsymbol{x})=f\left(x_{i}, \boldsymbol{x}_{-i}\right)=f\left(t, \boldsymbol{x}_{-i}\right)+\left(x_{i}-t\right) \frac{\partial f}{\partial x_{i}}\left(c, \boldsymbol{x}_{-i}\right)
$$

for a $c$ between $x_{i}$ and $t$, thus $c \in[t-h / 2, t+h / 2]$. Replacing by random variables, we get

$$
f\left(X_{i}^{h}, \boldsymbol{X}_{-i}\right)=f\left(t, \boldsymbol{X}_{-i}\right)+\left(X_{i}^{h}-t\right) \frac{\partial f}{\partial x_{i}}\left(C, \boldsymbol{X}_{-i}\right),
$$

where $C$ is a random variable such that $C \in[t-h / 2, t+h / 2]$. Denoting $Q=B_{i} \frac{\partial f}{\partial x_{i}}\left(C, \boldsymbol{X}_{-i}\right)$, we then have

$$
f\left(X_{i}^{h}, \boldsymbol{X}_{-i}\right)=f\left(t, \boldsymbol{X}_{-i}\right)+h Q .
$$

Now remark that $Q$ is bounded since both $B_{i}$ and $\frac{\partial f}{\partial x_{i}}$ are bounded (by continuity of $\frac{\partial f}{\partial x_{i}}$ on the compact set $\left.[0,1]^{d}\right)$. This implies that $\mathrm{E}\left(f\left(X_{i}^{h}, \boldsymbol{X}_{-i}\right)\right) \stackrel{h \rightarrow 0}{\longrightarrow}$ $\mathrm{E}\left(f\left(t, \boldsymbol{X}_{-i}\right)\right)$ by Lebesgue theorem as well as $\mathrm{E}\left(f\left(X_{i}^{h}, \boldsymbol{X}_{-i}\right)^{2}\right) \stackrel{h \rightarrow 0}{\longrightarrow} \mathrm{E}\left(f\left(t, \boldsymbol{X}_{-i}\right)^{2}\right)$. The result follows.

Proof of the second statement The proof is similar to the one of 1 . Write the Taylor-Lagrange expansion of $f$ between a real number $x_{i} \in[t-h / 2, t+$ $h / 2] \subseteq[0,1]$ and $t$

$$
f(\boldsymbol{x})=f\left(x_{i}, \boldsymbol{x}_{-i}\right)=f\left(t, \boldsymbol{x}_{-i}\right)+\left(x_{i}-t\right) \frac{\partial f}{\partial x_{i}}\left(t, \boldsymbol{x}_{-i}\right)+\frac{1}{2}\left(x_{i}-t\right)^{2} \frac{\partial^{2} f}{\partial x_{i}^{2}}\left(c, \boldsymbol{x}_{-i}\right)
$$

for a $c$ between $x_{i}$ and $t$, thus $c \in[t-h / 2, t+h / 2]$. Replacing by random variables, we have

$$
f\left(X_{i}^{h}, \boldsymbol{X}_{-i}\right)=f\left(t, \boldsymbol{X}_{-i}\right)+h B_{i} \frac{\partial f}{\partial x_{i}}\left(t, \boldsymbol{X}_{-i}\right)+h^{2} \widetilde{R}
$$

with $\widetilde{R}=\frac{1}{2} B_{i}^{2} \frac{\partial^{2} f}{\partial x_{i}^{2}}\left(C, \boldsymbol{X}_{-i}\right)$, where $C$ is a random variable, $C \in[t-h / 2, t+$ $h / 2]$. Then, using the independence between $X_{i}^{h}$ and $\boldsymbol{X}_{-i}$, we have

$$
\mathrm{E}\left[f\left(X_{i}^{h}, \boldsymbol{X}_{-i}\right) \mid X_{i}^{h}\right]=\beta_{0}+h \beta_{1} B_{i}+h^{2} R
$$


with $\beta_{0}=\mathrm{E}\left(f\left(t, \boldsymbol{X}_{-i}\right)\right), \beta_{1}=\mathrm{E}\left(\frac{\partial f}{\partial x_{i}}\left(t, \boldsymbol{X}_{-i}\right)\right)$, and $R=\mathrm{E}\left[\widetilde{R} \mid X_{i}^{h}\right]$. Notice that $\beta_{1}=\mathrm{E}\left[\frac{\partial f}{\partial x_{i}}(\mathbf{X}) \mid X_{i}=t\right]$, by using that $X_{i}$ and $\boldsymbol{X}_{-i}$ are independent. Finally

$$
\operatorname{var}\left(\mathrm{E}\left[f\left(X_{i}^{h}, \boldsymbol{X}_{-i}\right) \mid X_{i}^{h}\right]\right)=h^{2} \beta_{1}^{2} \operatorname{var}\left(B_{i}\right)+2 h^{3} \beta_{1} \operatorname{Cov}\left(B_{i}, R\right)+h^{4} \operatorname{var}(R) .
$$

Now remark that $\widetilde{R}$ is a bounded random variable by continuity of $\frac{\partial^{2} f}{\partial x_{i}^{2}}$ on the compact set $[0,1]^{d}$, and thus $R$ is bounded as well. This implies that $\operatorname{var}(R)=O(1)$ and $\operatorname{Cov}\left(B_{i}, R\right)=O(1)$, and the result follows.

Proof of the third statement With the same notations as in the proof of 2 ., we have

$$
\mathrm{E}\left[f\left(X_{i}^{h}, \boldsymbol{X}_{-i}\right) \mid \boldsymbol{X}_{-i}\right]=f\left(t, \boldsymbol{X}_{-i}\right)+h \mathrm{E}\left(B_{i}\right) \frac{\partial f}{\partial x_{i}}\left(t, \boldsymbol{X}_{-i}\right)+h^{2} \mathrm{E}\left[\widetilde{R} \mid \boldsymbol{X}_{-i}\right]
$$

and thus

$$
f\left(X_{i}^{h}, \boldsymbol{X}_{-i}\right)-\mathrm{E}\left[f\left(X_{i}^{h}, \boldsymbol{X}_{-i}\right) \mid \boldsymbol{X}_{-i}\right]=h\left(B_{i}-\mathrm{E}\left(B_{i}\right)\right) \frac{\partial f}{\partial x_{i}}\left(t, \boldsymbol{X}_{-i}\right)+h^{2} S
$$

with $S=\widetilde{R}-\mathrm{E}\left[\widetilde{R} \mid \boldsymbol{X}_{-i}\right]$. Since $S$ is centered, we have by independence of $B_{i}$ and $\frac{\partial f}{\partial x_{i}}\left(t, \boldsymbol{X}_{-i}\right)$

$$
\begin{aligned}
& \mathrm{E}\left(\left(f\left(X_{i}^{h}, \boldsymbol{X}_{-i}\right)-\mathrm{E}\left[f\left(X_{i}^{h}, \boldsymbol{X}_{-i}\right) \mid \boldsymbol{X}_{-i}\right]\right)^{2}\right)= \\
& \beta_{2} \operatorname{var}\left(B_{i}\right) h^{2}+2 h^{3} \operatorname{Cov}\left(\left(B_{i}-\mathrm{E}\left(B_{i}\right)\right) \frac{\partial f}{\partial x_{i}}\left(t, \boldsymbol{X}_{-i}\right), S\right)+h^{4} \operatorname{var}(S),
\end{aligned}
$$

with $\beta_{2}=\mathrm{E}\left(\left(\frac{\partial f}{\partial x_{i}}\left(t, \boldsymbol{X}_{-i}\right)\right)^{2}\right)$. Notice that $\beta_{2}=\mathrm{E}\left[\left(\frac{\partial f}{\partial x_{i}}(\mathbf{X})\right)^{2} \mid X_{i}=t\right]$, by using the independence between $X_{i}$ and $\boldsymbol{X}_{-i}$. The result follows in the same way as in the proof of 2 . by using that $\frac{\partial f}{\partial x_{i}}\left(t, \boldsymbol{X}_{-i}\right)$ and $S$ are bounded random variables.

Proof of Proposition 2. The first point is obtained directly by writing

$$
\mathrm{E}\left[\zeta_{i}^{T}\left(X_{i}\right)\right]=\mathrm{E}\left[\mathrm{E}\left(\left(\frac{\partial f}{\partial x_{i}}\left(X_{i}, \mathbf{X}_{-i}\right)\right)^{2} \mid X_{i}\right)\right]=\nu_{i}
$$


For the second point, compute the first-order Sobol' index of $\frac{\partial f}{\partial x_{i}}($.$) corre-$ sponding to the input variable $X_{i}$ by using the pick-freeze formula (Sobol', 1993) with $\mathbf{Z}_{-i}$ an independent copy of $\mathbf{X}_{i}$,

$$
D_{i}\left(\frac{\partial f}{\partial x_{i}}(\mathbf{X})\right)=\mathrm{E}\left[\frac{\partial f}{\partial x_{i}}\left(X_{i}, \mathbf{X}_{-\mathbf{i}}\right) \frac{\partial f}{\partial x_{i}}\left(X_{i}, \mathbf{Z}_{-i}\right)\right]-\left(\left(\frac{\partial f}{\partial x_{i}}\right)_{0}\right)^{2} .
$$

where $\left(\frac{\partial f}{\partial x_{i}}\right)_{0}=E\left(\frac{\partial f}{\partial x_{i}}(\mathbf{X})\right)$. As both factors in the expectation are independent conditionally on $X_{i}$, we can write

$$
\begin{aligned}
D_{i}\left(\frac{\partial f}{\partial x_{i}}(\mathbf{X})\right) & =\mathrm{E}\left[\mathrm{E}\left(\frac{\partial f}{\partial x_{i}}\left(X_{i}, \mathbf{X}_{-\mathbf{i}}\right) \frac{\partial f}{\partial x_{i}}\left(X_{i}, \mathbf{Z}_{-i}\right) \mid X_{i}\right)\right]-\left(\left(\frac{\partial f}{\partial x_{i}}\right)_{0}\right)^{2} \\
& =\mathrm{E}\left[\left(\mathrm{E}\left[\frac{\partial f}{\partial x_{i}}\left(X_{i}, \mathbf{X}_{-i}\right) \mid X_{i}\right]\right)\left(\mathrm{E}\left[\frac{\partial f}{\partial x_{i}}\left(X_{i}, \mathbf{Z}_{-i}\right) \mid X_{i}\right]\right)\right]-\left(\left(\frac{\partial f}{\partial x_{i}}\right)_{0}\right)^{2},
\end{aligned}
$$

and due to the same distribution of $\mathbf{X}_{-i}$ and $\mathbf{Z}_{-i}$, we obtain

$$
D_{i}\left(\frac{\partial f}{\partial x_{i}}(\mathbf{X})\right)=\mathrm{E}\left[\left(\mathrm{E}\left[\frac{\partial f}{\partial x_{i}}\left(X_{i}, \mathbf{X}_{-i}\right) \mid X_{i}\right]\right)^{2}\right]-\left(\left(\frac{\partial f}{\partial x_{i}}\right)_{0}\right)^{2}
$$

\section{References}

Fang, K., Li, R., Sudjianto, A., 2006. Design and modeling for computer experiments. Chapman \& Hall/CRC, London.

Fruth, J., Roustant, O., Kuhnt, S., 2014. Total interaction index: A variance-based sensitivity index for second-order interaction screening. Journal of Statistical Planning and Inference 147, $212-223$.

Hastie, T., Tibshirani, R.J., 1990. Generalized additive models. Chapman \& Hall/CR, London.

Hoeffding, W., 1948. A class of statistics with asymptotically normal distribution. The Annals of Mathematical Statistics 19, 293-325.

Homma, T., Saltelli, A., 1996. Importance measures in global sensitivity analysis of nonlinear models. Reliability Engineering \& System Safety 52, 1-17.

Iooss, B., Popelin, A.L., Blatman, G., Ciric, C., Gamboa, F., Lacaze, S., Lam, M., 2012. Some new insights in derivative-based global sensitivity measures. PSAM 11 \& ESREL 2012 Conference, Helsinki, Finland . 
Ishigami, T., Homma, T., 1990. An importance quantification technique in uncertainty analysis for computer models, in: Ayyub, B.M. (Ed.), Proceedings of the ISUMA '90, pp. 398-403.

Kucherenko, S., Rodriguez-Fernandez, M., Pantelides, C., Shah, N., 2009. Monte Carlo evaluation of derivative-based global sensitivity measures. Reliability Engineering \& System Safety 94, $1135-1148$.

Lamboni, M., Iooss, B., Popelin, A.L., Gamboa, F., 2013. Derivative-based global sensitivity measures: general links with Sobol' indices and numerical tests. Mathematics and Computers in Simulation 87, 45-54.

Li, L., Lu, Z., Hu, J., 2014. A new kind of regional importance measure of the input variable and its state dependent parameter solution. Reliability Engineering \& System Safety 128, 1-16.

Lu, Z., 1996. Multivariate locally weighted polynomial fitting and partial derivative estimation. Journal of Multivariate Analysis 59, 187-205.

McKay, M.D., Beckman, R.J., Conover, W.J., 1979. A comparison of three methods for selecting values of input variables in the analysis of output from a computer code. Communications in Statistics - Theory and Methods 21, 239-245.

Mühlenstädt, T., Roustant, O., Carraro, L., Kuhnt, S., 2012. Data-driven Kriging models based on FANOVA-decomposition. Statistics and Computing 22, 723-738.

Pannier, S., Graf, W., 2015. Sectional global sensitivity measures. Reliability Engineering \& System Safety 134, 110-117.

Pujol, G., Iooss, B., with contributions from Paul Lemaitre, A.J., Gilquin, L., Gratiet, L.L., Touati, T., Ramos, B., Fruth, J., Veiga, S.D., 2014. sensitivity: Sensitivity Analysis. URL: http://CRAN.R-project.org/package=sensitivity. r package version 1.10.1.

Rakovec, O., Hill, M.C., Clark, M.P., Weerts, A.H., Teuling, A.J., Uijlenhoet, R., 2014. Distributed evaluation of local sensitivity analysis (delsa), with application to hydrologic models. Water Resources Research 50, 409-426.

Roustant, O., Fruth, J., Iooss, B., Kuhnt, S., 2014. Crossed-derivative based sensitivity measures for interaction screening. Mathematics and Computers in Simulation 105, 105-118.

Roustant, O., Ginsbourger, D., Deville, Y., 2012. DiceKriging, DiceOptim: Two R packages for the analysis of computer experiments by Kriging-based metamodeling and optimization. Journal of Statistical Software 51, 1-55. 
Saltelli, A., 2002. Making best use of model evaluations to compute sensitivity indices. Computer Physics Communications 145, 280-297.

Saltelli, A., Chan, K., Scott, E., 2000. Sensitivity analysis. Wiley, Chichester.

Santner, T., Williams, B., Notz, W., 2003. The design and analysis of computer experiments. Springer, New York.

Sobol', I., Gershman, A., 1995. On an alternative global sensitivity estimators, in: Proceedings of SAMO 1995, Belgirate. pp. 40-42.

Sobol', I.M., 1993. Sensitivity estimates for non linear mathematical models. Mathematical Modelling and Computational Experiments 1, 407-414.

Touzani, S., Busby, D., 2014. Screening method using the derivative-based global sensitivity indices with application to reservoir simulator. Oil \& Gas Science and Technology - Revue d'IFP Energies nouvelles 69, 619-632.

Turanyi, T., 1990. Sensitivity analysis of complex kinetic systems. tools and applications. J. Math. Chem. 5, 203-248.

ul Hassan, H., Fruth, J., Güner, A., Tekkaya, A.E., 2013. Finite element simulations for sheet metal forming process with functional input for the minimization of springback, in: IDDRG conference 2013, Zrich. pp. 393-398.

Wei, P., Lu, Z., Ruan, W., Song, J., 2014. Regional sensitivity analysis using revised mean and variance ratio functions. Reliability Engineering \& System Safety 121, 121-135.

Welch, W.J., Buck, R.J., Sacks, J., Wynn, H.P., Mitchell, T.J., Morris, M.D., 1992. Screening, predicting, and computer experiments. Communications in Statistics - Theory and Methods $34,15-25$. 

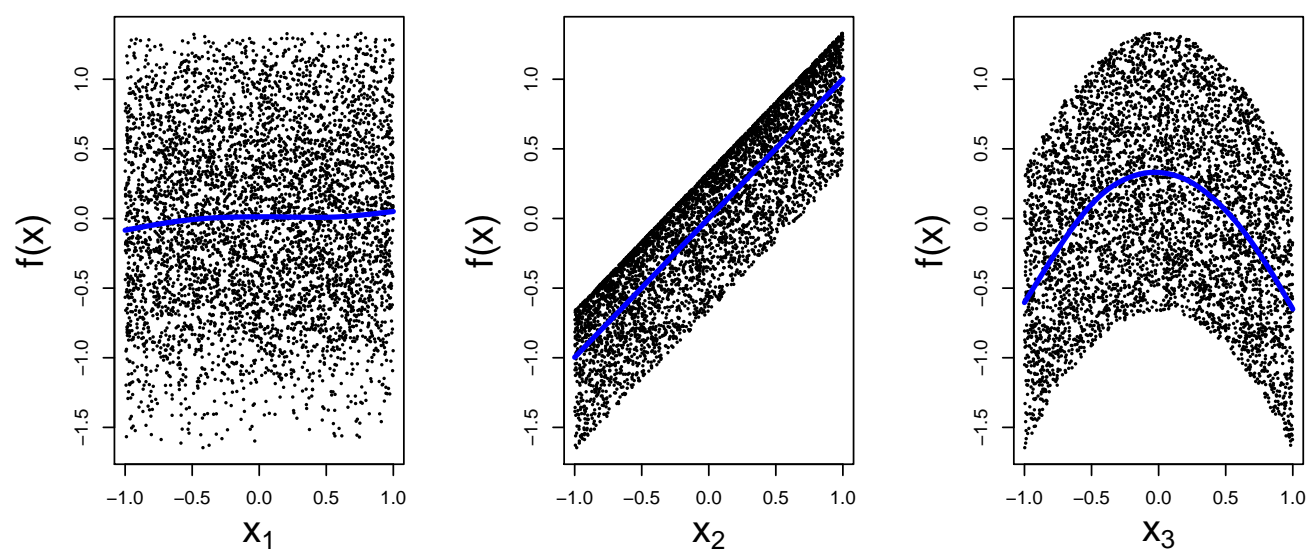

Figure 1: Scatterplots of the polynomial function and main effects estimated by smoothing. A 5000 -point sample was used. The global mean has been removed. 

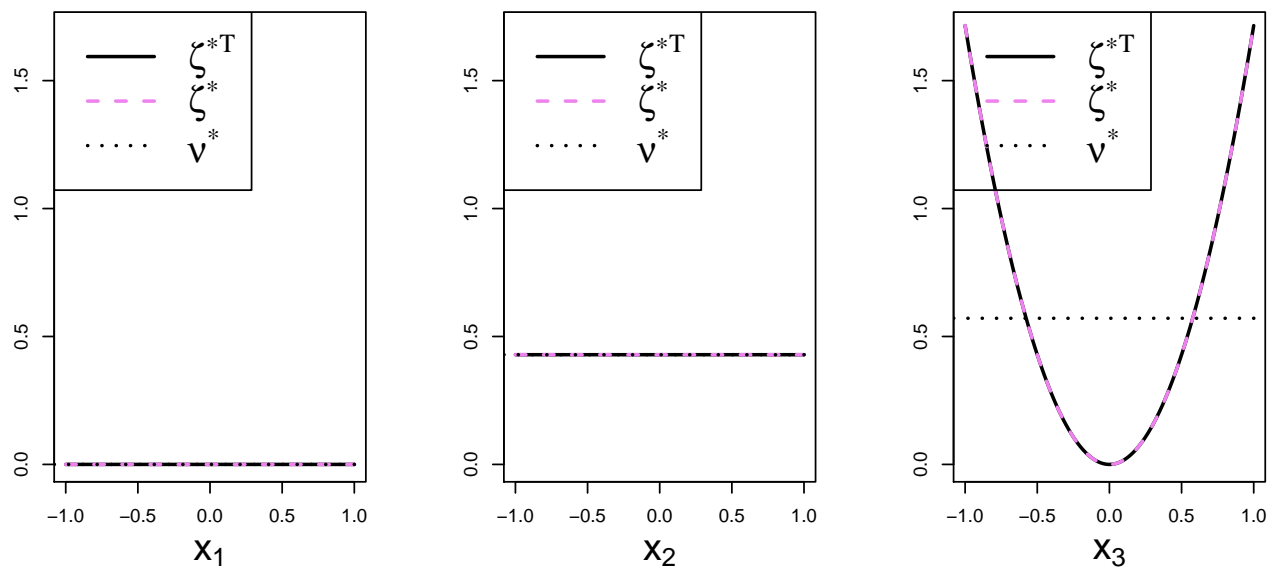

Figure 2: Support analysis of the polynomial function. Estimated curves are normalized by the sum of DGSM values. A 5000-point sample was used. 

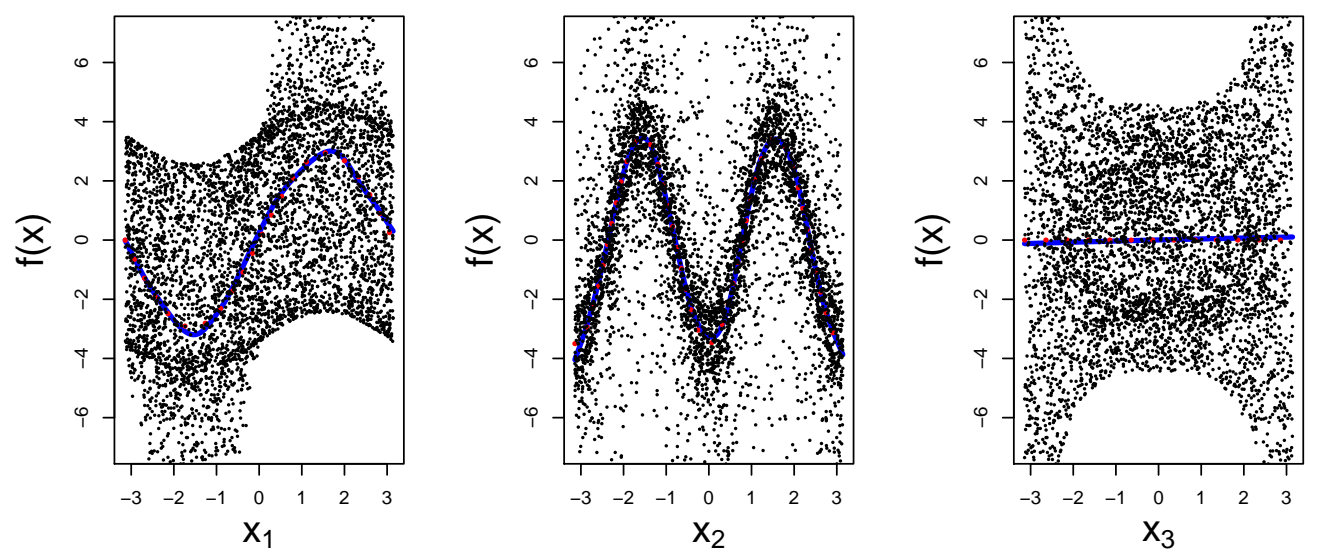

Figure 3: Scatterplots and main effects of the Ishigami function, estimated by smoothing from a 5000-point sample. The global mean has been removed. Theoretical curves are superimposed in dotted lines. 

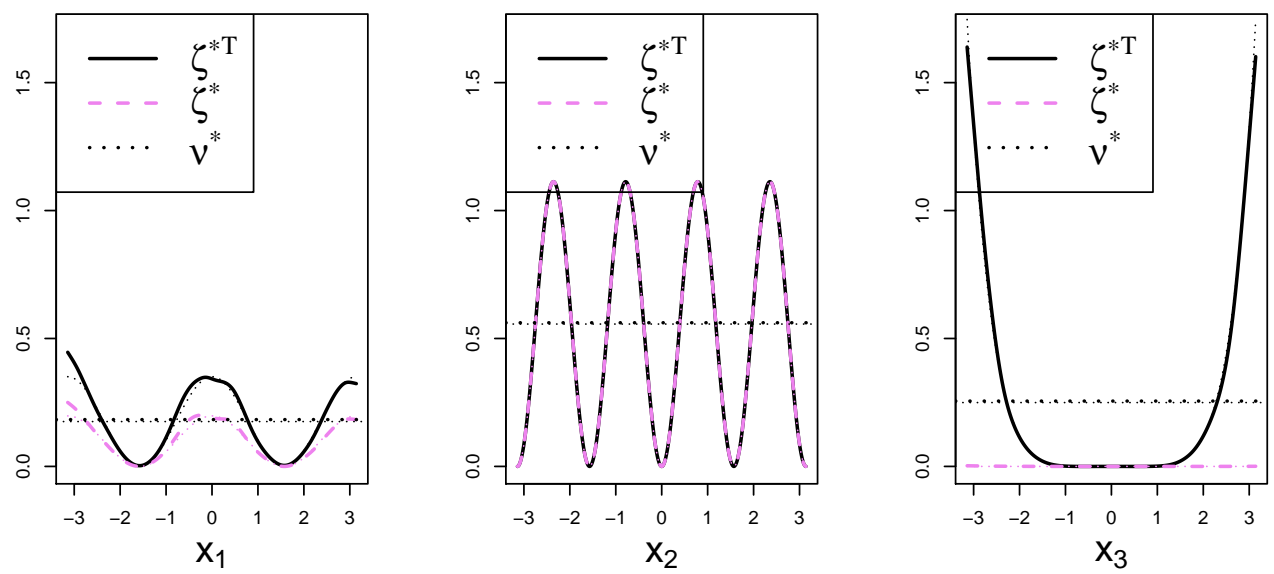

Figure 4: Support analysis of the Ishigami function. Estimated curves are computed on a 5000-point sample and normalized by the sum of DGSM values. Theoretical curves are superimposed in thinner lines. 

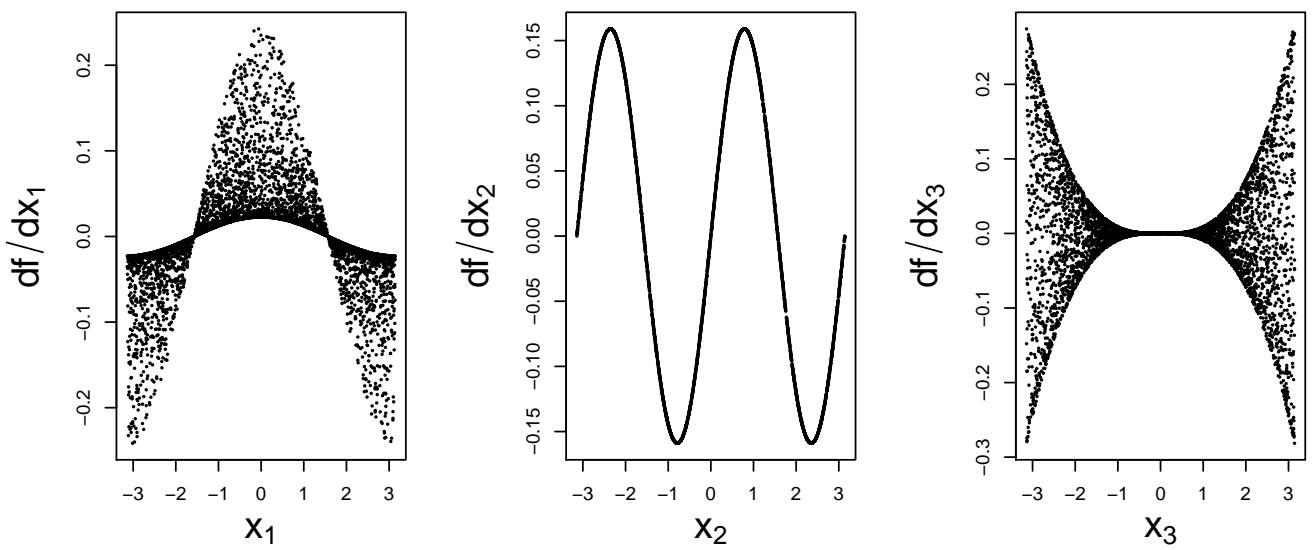

Figure 5: Diagonal scatterplots $\left(X_{i}, \frac{\partial f(\mathbf{X})}{\partial x_{i}}\right)$ of the gradient of Ishigami function, for a 5000 -point sample. 

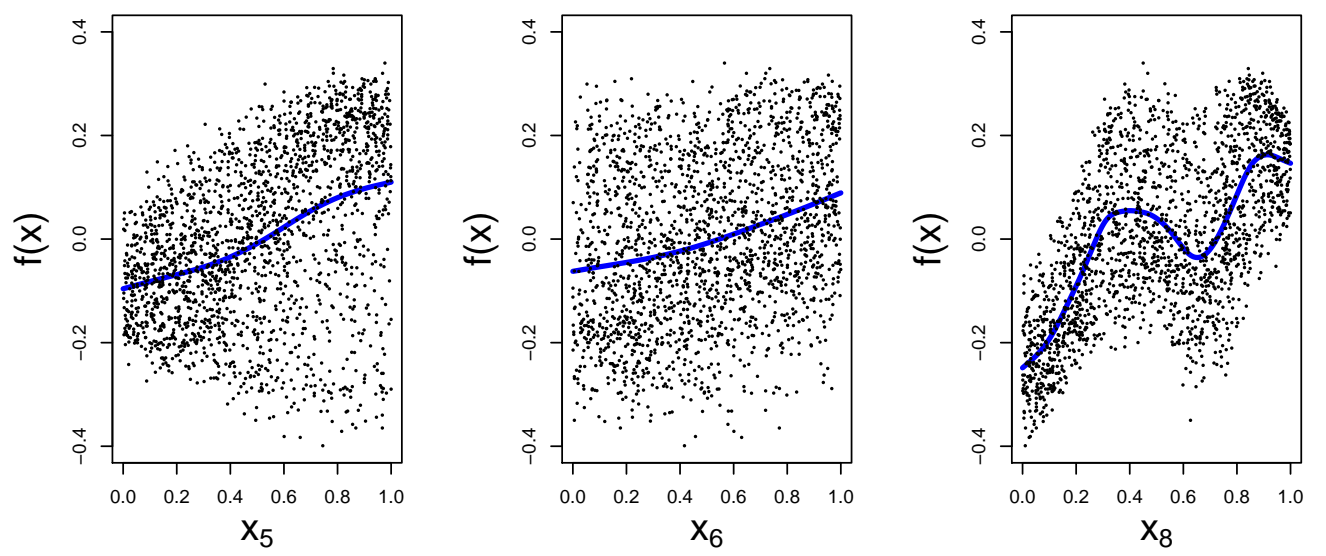

Figure 6: Scatterplots and estimated main effects for the GP metamodel in the sheet metal application. A 2000 -point sample was used. The global mean has been removed. 

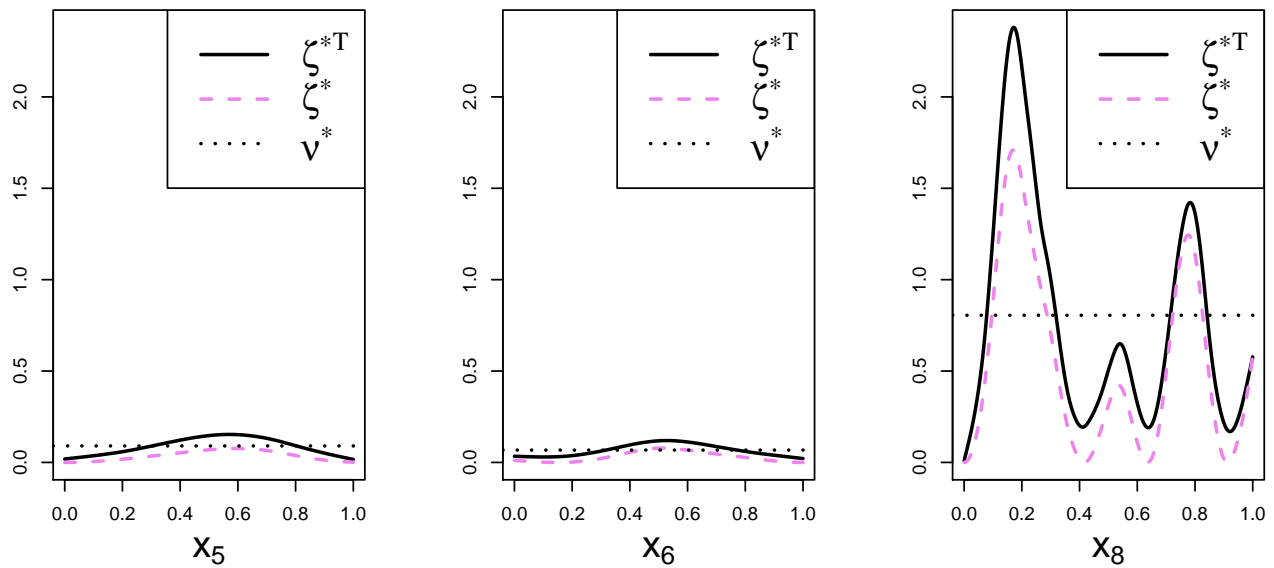

Figure 7: Support analysis of the sheet metal application. Estimated curves are computed on a 2000 -point sample and normalized by the sum of DGSM values. 


\begin{tabular}{ccccc}
\hline & Sobol' index & total Sobol' index & DGSM & normalized DGSM \\
\hline$X_{1}$ & 0.314 & 0.558 & 7.720 & 0.179 \\
$X_{2}$ & 0.442 & 0.442 & 24.5 & 0.567 \\
$X_{3}$ & 0 & 0.244 & 10.987 & 0.254 \\
\hline
\end{tabular}

Table 1: Global sensitivity values for Ishigami function. 
Table 2: Input variables of the thickness reduction application.

\begin{tabular}{|lll|}
\hline & input variable & feasible region \\
\hline$X_{1}$ & flow stress & $100-200 \mathrm{MPa}$ \\
$X_{2}$ & initial sheet thickness & $0.5-1.7 \mathrm{~mm}$ \\
$X_{3}$ & blankholder force & $50-200 \mathrm{kN}$ \\
$X_{4}$ & friction; $1^{\text {st }}$ third of process time & $0-0.14$ \\
$X_{5}$ & friction: 2 \\
$X_{6}$ & friction: $3^{\text {rd }}$ third of process time & $0-0.14$ \\
$X_{7}$ & hardening exponent & $0-0.14$ \\
$X_{8}$ & sheet layout & $0.1-0.3$ \\
\hline
\end{tabular}




\begin{tabular}{ccccc}
\hline & Sobol' index & total Sobol' index & DGSM & normalized DGSM \\
\hline$X_{5}$ & 0.136 & 0.301 & 0.087 & 0.090 \\
$X_{6}$ & 0.095 & 0.205 & 0.065 & 0.068 \\
$X_{8}$ & 0.470 & 0.692 & 0.776 & 0.805 \\
\hline
\end{tabular}

Table 3: Global sensitivity values of the GP metamodel for the sheet metal application, limited to three most influential variables. 